\title{
Optic Disc
}

National Cancer Institute

\section{Source}

National Cancer Institute. Optic Disc. NCI Thesaurus. Code C12760.

A portion of the retina at which the axons of the ganglion cells exit the eyeball to form

the optic nerve. No light-sensitive photoreceptors are contained within this portion of the retina. 